TRANSACTIONS OF THE

AMERICAN MATHEMATICAL SOCIETY

Volume 350, Number 9, September 1998, Pages 3523-3535

S 0002-9947(98)02089-3

\title{
PERIODIC BILLIARD ORBITS ARE DENSE IN RATIONAL POLYGONS
}

\author{
M. BOSHERNITZAN, G. GALPERIN, T. KRÜGER, AND S. TROUBETZKOY
}

\begin{abstract}
We show that periodic orbits are dense in the phase space for billiards in polygons for which the angle between each pair of sides is a rational multiple of $\pi$.
\end{abstract}

\section{INTRODUCTION}

A billiard ball, i.e. a point mass, moves inside a polygon $Q$ with unit speed along a straight line until it reaches the boundary $\partial Q$ of the polygon, then instantaneously changes direction according to the mirror law: "the angle of incidence is equal to the angle of reflection," and continues along the new line (Fig. 1(a)). Despite the simplicity of this description there is much that is unknown about the existence and the description of periodic orbits in arbitrary polygons. On the other hand, quite a bit is known about a special class of polygons, namely, rational polygons. A polygon is called rational if the angle between each pair of sides is a rational multiple of $\pi$. The main theorem we will prove is

Theorem 1. For rational polygons, periodic points of the billiard flow are dense in the phase space $\mathbf{M}$ of the billiard flow.

Theorem 1 is a strengthening of Masur's theorem [M], which says that any rational polygon has "many" periodic billiard trajectories; more precisely, the set of directions of the periodic trajectories is dense in the set of velocity directions $\mathbf{S}^{1}$. We will also prove some refinements of Theorem 1: the "well distribution" of periodic orbits in the polygon and the residuality of the points $q \in Q$ with a dense set of periodic directions (precise statements will be given in section 3 ).

The structure of the article is as follows. In section 2 we give a brief description of billiards in polygons and some results related to Theorem 1. In section 3 we state the strengthenings of Theorem 1, and the proofs will be given in section 4 .

\section{DESCRIPTION OF BILLIARDS IN POLYGONS}

The trace of a moving billiard ball is called a billiard trajectory or orbit. If a billiard trajectory hits a vertex of the polygon, then it is called singular. For convenience we will define such billiard trajectories by continuity from the left (with respect to a fixed orientation of the boundary); thus every trajectory is infinite and

Received by the editors July 29, 1996.

1991 Mathematics Subject Classification. Primary 58F05.

MB is partially supported by NSF-DMS-9224667.

GG thanks the Alexander von Humboldt Stiftung for their support.

ST thanks the Deutsche Forschungsgemeinschaft for their support.

(C)1998 American Mathematical Society 


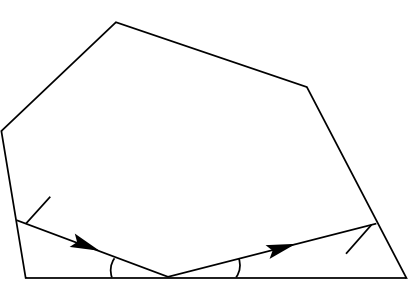

(a)

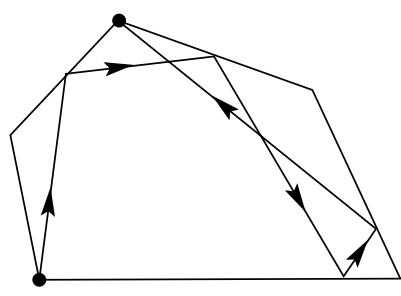

(b)

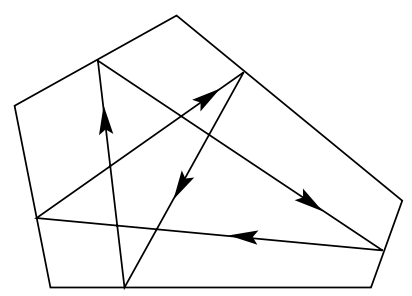

(c)

Figure 1. (a) The mirror law, (b) a generalized diagonal, (c) a periodic orbit

the singular trajectories are the discontinuities of the flow. It is convenient to consider the set of singular trajectory segments which start at a vertex and end at a vertex. Such segments are called generalized diagonals and the number of links is called the length of the generalized diagonal (Fig. 1(b)). We remark that the set of generalized diagonals is countable. If a billiard ball returns to its initial position and has the same velocity direction, then its orbit is called periodic (Fig. 1(c)).

There is a very useful tool in the analysis of billiards in polygons: the unfolding of trajectories. Instead of reflecting the trajectory with respect to the side of the polygon it hits we reflect the polygon itself with respect to the same side. The two adjacent links of the trajectory then become part of a straight line (Fig. 2(a)). Continuing this procedure forever unfolds the trajectory into a half-line through a forward corridor of polygons. The backward trajectory can be similarly unfolded. We enumerate the unfolded polygons starting with zero, and the length of an unfolding is the number of polygons it contains (Fig. 2(b)).

The set of pairs $\{(q, v)\}$, where $q \in Q$ is a position of the ball and $v \in \mathbf{S}^{1}$ is its velocity, constitutes the phase space $\mathbf{M}$ of the billiard flow. The phase space $\mathbf{M}$ can be thought of as a right prism foliated with a collection of "floors" $Q(\varphi):=Q \times \varphi$ with $\varphi \in \mathbf{S}^{1}$. Then the billiard flow in the phase space can be imagined as a straight line flow on the floors of the phase space. When the flow reaches the boundary it jumps from the point $(q, \varphi)$ to the point $\left(q, \varphi^{\prime}\right)$ on the floor $Q\left(\varphi^{\prime}\right)$, where $\varphi$ and $\varphi^{\prime}$ are related by the mirror law (Fig. 3). In case the polygon is rational, the flow is restricted to a finite number of floors. Using the identifications of the boundary one can glue these floors together to produce an orientable surface $R_{\varphi}$ (see the survey article $[\mathrm{Gu}]$ for a full explanation). 

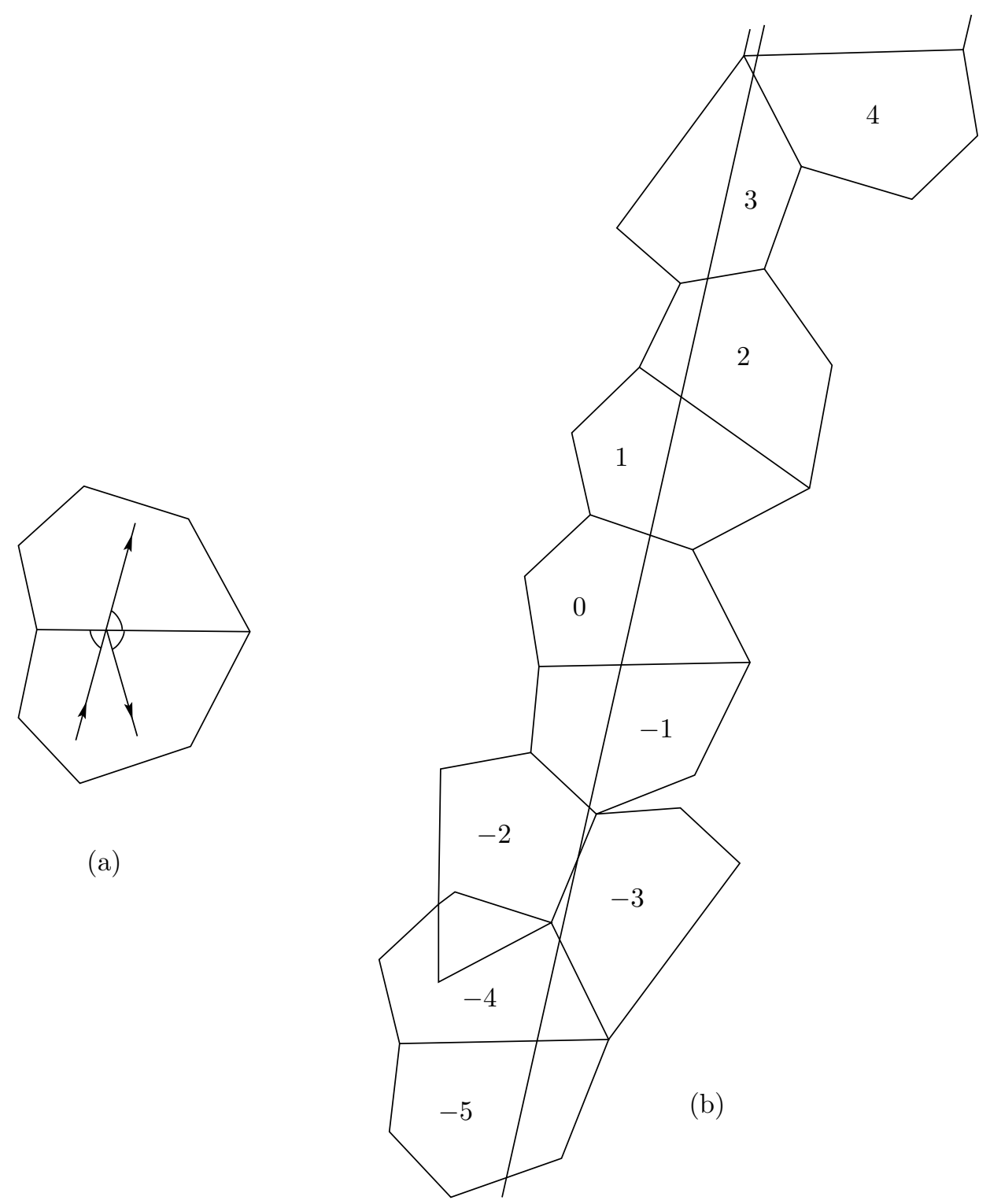

FiguRE 2. Unfolding a trajectory

For any $\varphi \in S^{1}$ for which $R_{\varphi}$ contains no generalized diagonal, the flow $\phi_{t}$ restricted to the invariant surface $R_{\varphi}$ is minimal, i.e. the orbit of every point is dense $[\mathrm{Gu}]$. Using Teichmüller theory Kerckhoff, Masur, and Smillie showed that for almost every $\varphi \in \mathbf{S}^{1}$ the flow $\left.\phi_{t}\right|_{R_{\varphi}}$ is uniquely ergodic, i.e. the only ergodic invariant measure is the Lebesgue measure [KMS]. Using similar techniques, Masur has shown that for a dense set of directions $\varphi \in S^{1}$ the flow $\phi_{t}$ restricted to $R_{\varphi}$ has at least one periodic point $[\mathrm{M}]$. However, his result gives no indication of the location of the periodic orbit on the invariant surface $R_{\varphi}$. 


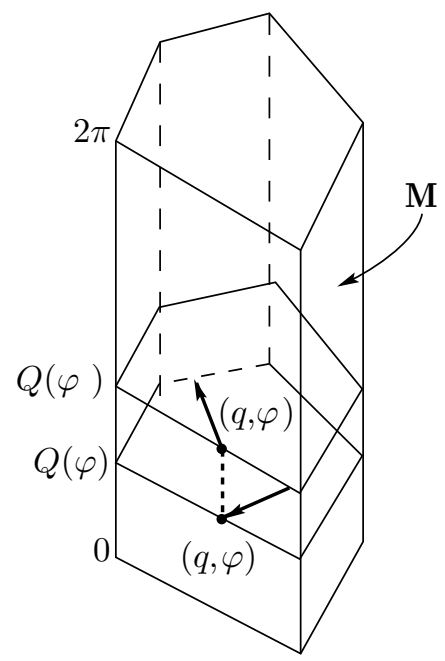

Figure 3. The mirror law in phase space

\section{REsults}

Throughout this section we will assume that $Q$ is a rational polygon.

Theorem 2. Periodic points of the billiard flow are dense in the phase space $\mathbf{M}$.

We will also prove a slightly stronger theorem. For this purpose we need one more definition. A periodic orbit $\gamma$ is called $\varepsilon$-well distributed on the table if for every convex set $A \subset Q$

$$
\left|\frac{\text { length }(\gamma \cap A)}{\operatorname{length}(\gamma)}-\mu_{\mathrm{Leb}}(A)\right|<\varepsilon .
$$

Here the length of a periodic orbit is its geometric length, not the number of links in its trajectory.

Theorem 3. The set of $\varepsilon$-well distributed periodic points of the billiard flow is dense in the phase space $\mathbf{M}$ for every $\varepsilon>0$.

Let $V$ be the set of $Q$ 's vertices and

$$
\begin{aligned}
G:=\{q \in Q \backslash V: \forall \varepsilon>0, \text { for a dense set of directions } \varphi \\
(q, \varphi) \text { is an } \varepsilon \text {-well distributed periodic point }\} .
\end{aligned}
$$

As another refinement of Theorem 1 we have:

Theorem 3. $G$ is residual. ${ }^{1}$

The natural question arises: does $G=Q \backslash V$ for all rational polygons? Does $G=Q \backslash V \bmod (0)$ for all polygons?

Equality in the stronger sense holds for integrable polygons (polygons which tile the plane under their reflection group), almost integrable polygons [Gu1], Veech

\footnotetext{
${ }^{1} \mathrm{~A}$ set is called residual if it contains a dense $G_{\delta}$ set, i.e. a countable intersection of open dense sets.
} 
polygons which include regular polygons, and closely related polygons [V], [V1]. Next we consider

$$
B:=\{q \in Q \backslash V: \text { there is no direction } \varphi \text { for which }(q, \varphi) \text { is periodic }\} .
$$

Theorem 4. If $Q$ is rational and convex, then $B$ is contained in a finite union of segments. If $Q$ is a rational triangle, then $B$ is at most finite.

Finally we turn to the question: for which angles $\varphi$ is there at least one orbit which is not $\varepsilon$-dense? We show:

Theorem 5. The set of directions for which there exists a non- $\varepsilon$-dense orbit is an at most countable closed set for any $\varepsilon>0$. There are examples when this set is not finite.

\section{Proofs of theorems}

For a direction $\theta \in \mathbf{S}^{1}$, a point $x \in R_{\theta}$ and an integer $N \geq 1$, denote by $\operatorname{Orb}(x, \theta, N)$ the union of the first $N$ links of $x$ 's forward orbit. If $x$ 's forward orbit terminates too early (i.e., hits a vertex prior to the completion of the first $N$ reflections) we set, by definition, $\operatorname{Orb}(x, \theta, N)=\emptyset$. The following lemma is needed.

Lemma 6. Let $\theta \in \mathbf{S}^{1}$ be a minimal direction, and let $\varepsilon>0$ be given. Then, for a sufficiently large integer $N$, $\operatorname{Orb}(x, \theta, N)$ is $\varepsilon$-dense in $R_{\theta}$, for all $x \in R_{\theta}$ for which $\operatorname{Orb}(x, \theta, N) \neq \emptyset$.

This lemma is a special case of a general principle that in minimal dynamical systems (in the discrete case of compact metric spaces) all finite pieces of orbits become arbitrarily dense once they are long enough (see, e.g., [W, Thm. 6.19]). Since the flow can be viewed as continuous on the whole of $R_{\theta}$ only after an appropriate modification in the definition of $R_{\theta}$ (not to be described here), the applicability of this principle to our situation is not completely obvious, and we choose to sketch a proof of the lemma which does not refer to this principle.

Proof of Lemma 6. First one easily verifies (and it is well known, see [BKM] or, in slightly more detail, [Bo1]) that minimality of the flow on $R_{\theta}$ is equivalent to the minimality of the associated interval exchange transformation (iet) $T_{\theta}$ defined on the interval $X_{\theta}$ which is a finite union of suitably contracted copies of the sides of the polygon $Q$.

When an arbitrary iet $(X, T)$ is induced on a subinterval $J \subset X$, the resulting transformation is known to be a new iet $(J, S)$ (see $[\mathrm{K}]$ or $[\mathrm{CFS}, \mathrm{Ch} .5]$ ). It is easily seen that for some number $N=N(J)$

$$
\bigcup_{k=0}^{N-1} T^{-k}(J)=\bigcup_{k=0}^{\infty} T^{-k}(J) .
$$

Indeed, one takes $N$ to be the maximal return time for $J$; it coincides with the maximum of the return times taken over (the finite number of) the subintervals of $J$ exchanged by $S$.

Since we are supposing that this iet $(X, T)$ is moreover minimal, then every orbit is dense in $X$ and thus must hit the subinterval $J \subset X$. It follows that $X=\bigcup_{k=0}^{\infty} T^{-k}(J)$, and hence

$$
X=\bigcup_{k=0}^{N-1} T^{-k}(J) .
$$




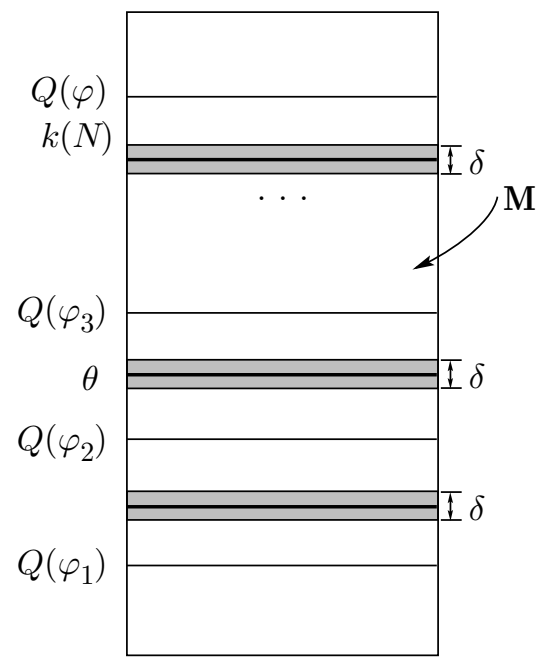

Figure 4 . The $\delta$-neighborhood of $R_{\theta}$ does not contain a generalized diagonal of length less than $2 N$

This last equality means that every orbit of length $N$ hits $J$. Since $J \subset X$ is arbitrary, it follows that sufficiently long (finite) orbits of a minimal iet become arbitrarily dense. This property of $T_{\theta}$ is easily extended to the initial flow on $R_{\theta}$, completing the proof of the lemma.

Proof of Theorem 1. In view of Lemma 6 , for an arbitrary minimal direction $\theta \in \mathbf{S}^{1}$ there exists an integer $N=N(\theta, \varepsilon)$ such that each of the sets

$$
\operatorname{Orb}^{+}(x)=\operatorname{Orb}(x, \theta, N) ; \operatorname{Orb}^{-}(x)=\operatorname{Orb}(x,-\theta, N)
$$

is either empty or $\varepsilon / 2$-dense in the surface $R_{\theta}$ (for notation, see the paragraph preceding Lemma 6). By avoiding a countable set of $\theta$ 's, we can assume that $R_{\theta}$ contains no generalized diagonals. There are only a finite number of generalized diagonals of length less than or equal to $2 N$. In the phase space $\mathbf{M}$ these generalized diagonals lie on a finite number of floors $Q\left(\varphi_{1}\right), Q\left(\varphi_{2}\right), \ldots, Q\left(\varphi_{k(N)}\right)$. Let $\delta:=\delta_{N}>$ 0 be so small that for any $\theta^{\prime}$ satisfying $\left|\theta-\theta^{\prime}\right|<\delta$ we have $Q\left(\varphi_{i}\right) \cap R_{\theta^{\prime}}=\emptyset$ for $i=1, \ldots, k(N)$ (Fig. 4). Therefore, if a generalized diagonal belongs to $R_{\theta^{\prime}}$, then its length is greater than $2 N$.

From Masur's theorem we know that there is a periodic point whose direction is arbitrarily close to $\theta$. In particular, we can find a periodic point $\left(q_{0}, \varphi_{0}\right) \in \mathbf{M}$ satisfying both $\left|\varphi_{0}-\theta\right|<\delta$ and $\left|\varphi_{0}-\theta\right|<\varepsilon /(2 \cdot \operatorname{const} \cdot N \cdot \operatorname{diam} Q)$. Here, const is a constant depending on $\theta$, which will be defined later. We consider the point $\left(q_{0}, \theta\right)$ and its forward unfolding of length $N$ and its backward unfolding of length $N$. We claim that either the corridor of length $N$ for the forward trajectory of $\left(q_{0}, \varphi_{0}\right)$ or the corridor of length $N$ for the backward trajectory of $\left(q_{0}, \varphi_{0}\right)$ coincides with the same length corridor of $\left(q_{0}, \theta\right)$.

Suppose this is not true. The forward corridors of $\left(q_{0}, \theta\right)$ and $\left(q_{0}, \varphi_{0}\right)$ coincide for a while. Define $j_{1}<N$ to be the length of the forward part of the corridors of $\left(q_{0}, \theta\right)$ and $\left(q_{0}, \varphi_{0}\right)$ that coincide. The two corridors "branch" apart at polygon number $j_{1}-1$; let $A$ be the common vertex of the branching corridors (or any one 


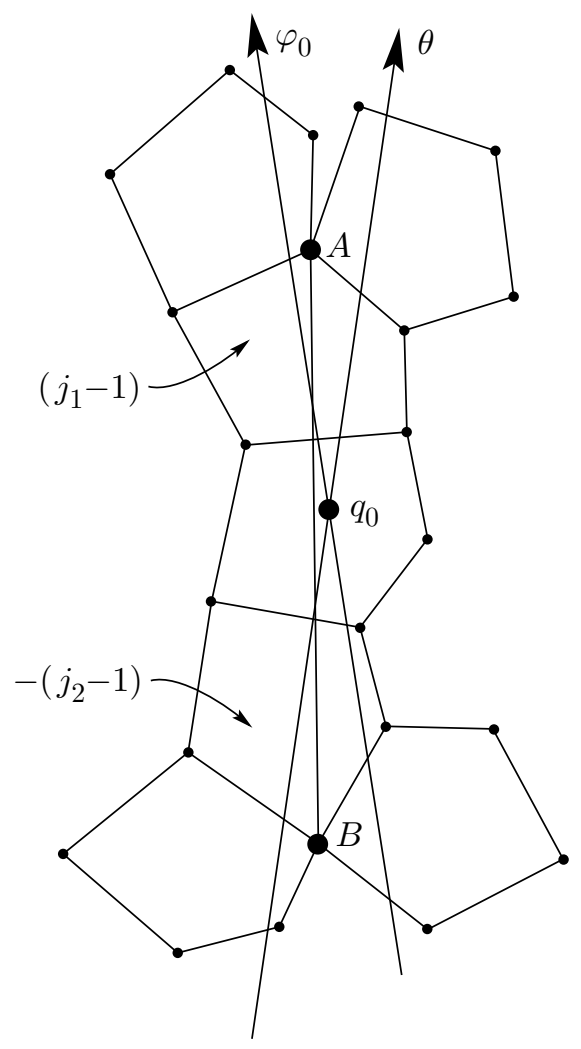

FIGURE 5a. Branching corridors

of the common vertices if there is more than one). Similarly, let $j_{2}<N$ be the length of the backward corridors and $B$ one of the common vertices of the backward branching corridors. There are two possibilities: either the straight line segment $A B$ lies entirely inside the corridor of $\left(q_{0}, \theta\right)$ and is therefore a generalized diagonal of length $j_{1}+j_{2}-1<2 N$, or it does not lie in the corridor entirely. If it lies in the corridor, then the direction of $A B$ lies in the interval of directions $\left\{t: \theta \leq t \leq \varphi_{0}\right\}$ (we assume $\varphi_{0}>\theta$ ) and thus is its distance to $\theta$ is less than $\delta$ (Fig. 5a). This contradicts the choice of $\delta$.

We now consider the second case, when the segment $A B$ does not lie entirely inside the corridor. Then there is at least one and at most finitely many vertices $C_{i}$ which separate the segment $A B$ from the corridor. Such vertices lie in the triangle $X q_{0} Y$ whose sides consist of a piece of $A B$ (the side $X Y$ ), a piece of the trajectory of $\left(q_{0}, \theta\right)$ (the side $\left.q_{0} Y\right)$ and a piece of the trajectory of $\left(q_{0}, \phi_{0}\right)$ (the side $\left.q_{0} X\right)$ (Fig. 5b). For each such vertex $C_{i}$ draw the segment $A C_{i}$. Consider the segment $A C_{i_{0}}$ which forms the smallest angle with the segment $A q_{0}$. If there is more than one such $C_{i_{0}}$, choose the one closest to $A$. Let $M, N$ be the points where the segment $A C_{i_{0}}$ meets the segments $X q_{0}$ and $Y q_{0}$ respectively. Then $C_{i_{0}}$ is a point on the segment $M N$. By assumption the triangle $M q_{0} N$ does not contain any vertices in its interior. Therefore $A C_{i_{0}}$ is a generalized diagonal of length less than 


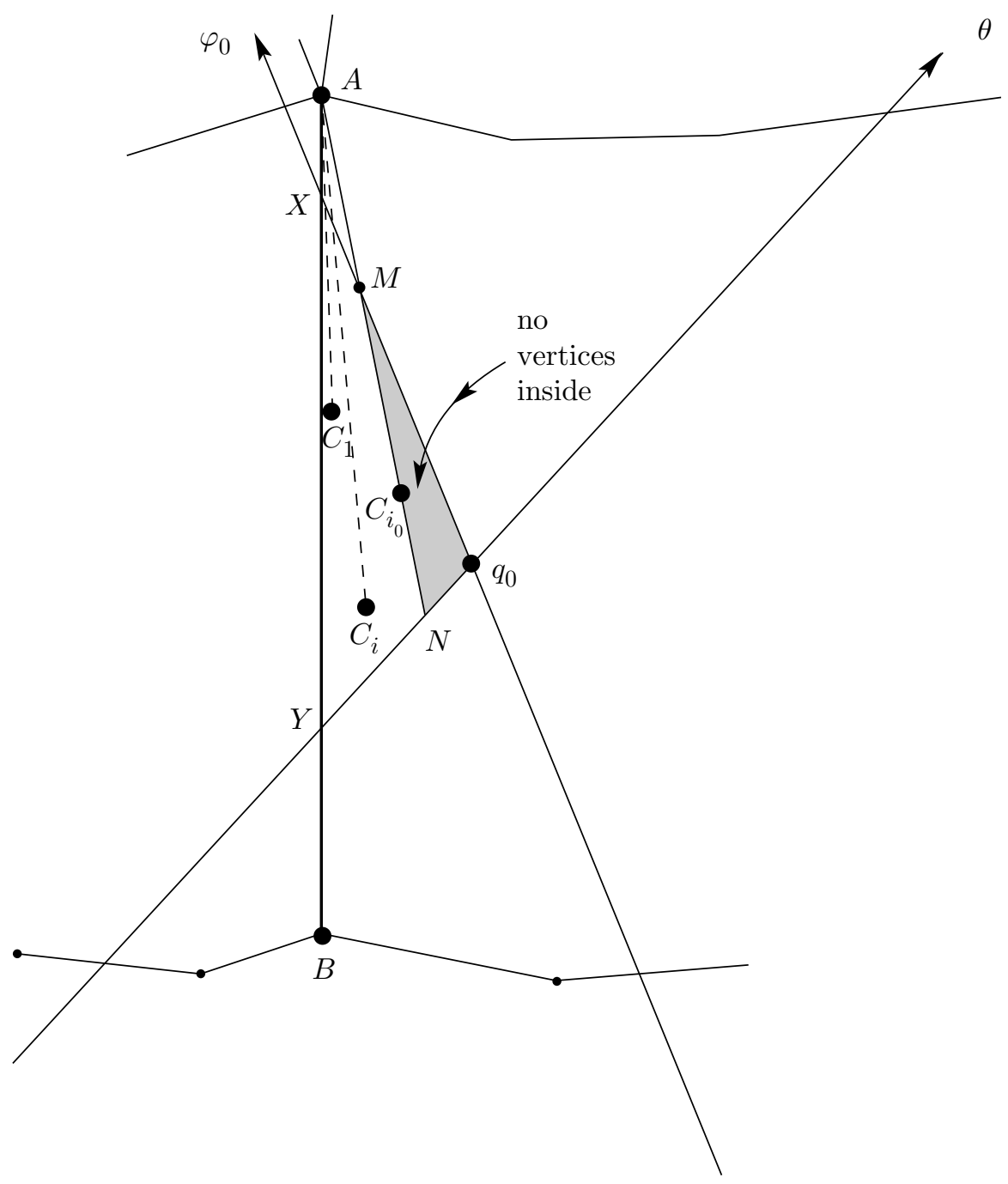

Figure 5b. Generalized diagonal $A C_{i_{0}}$

$j_{1}+j_{2}-1<2 N$. Furthermore the direction of $A C_{i_{0}}$ also lies between $\theta$ and $\phi_{0}$; thus we again have the same contradiction as before.

Without loss of generality we will assume that both the periodic trajectory and the dense trajectory stay in the same forward corridor. The endpoints of these two trajectories lie on the boundary of the $(N-1)$ st polygon. Since $\left|\varphi_{0}-\theta\right|<$ $\varepsilon /(2 \cdot \operatorname{const} \cdot N \cdot \operatorname{diam} Q)$, it follows that if the const is large enough, then the distance between the endpoints of the two unfolded trajectories is less than $\varepsilon / 2$ (Fig. 6). Furthermore, the first $N$ links of the dense trajectory are $\varepsilon / 2$-dense in $R_{\theta}$. These two facts combined show that the periodic trajectory is $\varepsilon$-dense in $R_{\varphi_{0}}$. This can be done for every minimal direction $\theta$. The set $\left\{R_{\theta}:\left.\phi\right|_{R_{\theta}}\right.$ is minimal $\}$ is dense in the whole phase space $\mathbf{M}$. Since $\varepsilon$ is arbitrary, this completes the proof of Theorem 1. 


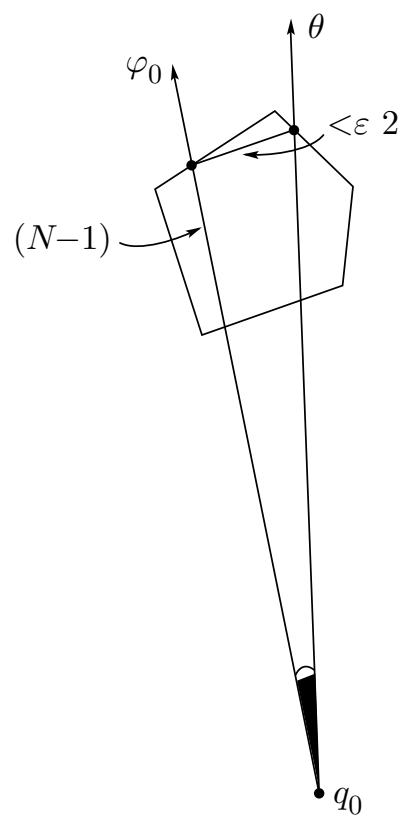

FiguRE 6. $\varepsilon / 2$-shadowing of length $N$

Proof of Theorem 2. We give an equivalent definition of $\varepsilon$-well distribution. We fix an embedding $Q \subset \mathbf{R}^{2}$. Let $A_{p, q, r, s}$ be the intersection of $Q$ with the open ball with center $(p / q, r / s)$ and diameter $1 /(\max (q, s))$. Enumerating gives rise to the countable basis $\left\{\tilde{A}_{i}\right\}$. A periodic orbit $\gamma$ is $\varepsilon$-well distributed on the table if for each set $\tilde{A}_{i}$ with $\operatorname{diam}\left(\tilde{A}_{i}\right)>\varepsilon$ we have

$$
\left|\frac{\operatorname{length}\left(\gamma \cap \tilde{A}_{i}\right)}{\operatorname{length}(\gamma)}-\mu_{\text {Leb }}\left(\tilde{A}_{i}\right)\right|<\varepsilon .
$$

For a fixed sufficiently small $\varepsilon>0$ for a convex set $A$ with sufficiently small diameter equation (1) automatically holds, and we can approximate any convex set $A$ with large diameter by finite unions and intersections of the $\tilde{A}_{j}$. Using this reasoning one can show that for every $\delta>0$ there is an $\varepsilon>0$ so that any $\varepsilon$-well distributed point in the sense above is $\delta$-well distributed in the sense of section 2 and $\varepsilon \rightarrow 0$ as $\delta \rightarrow 0$.

First of all we fix a uniquely ergodic direction $\theta \in \mathbf{S}^{1}$. As mentioned above, by avoiding a countable set of $\theta$ 's we can assume that $R_{\theta}$ contains no generalized diagonals. We claim that using the unique ergodicity we can choose $N$ so large that for all $x \in R_{\theta}$ the first $N$ links of $x$ 's forward orbit and the first $N$ links of $x$ 's backward orbit are both $\varepsilon / 3$-well distributed (the definition of well distributed is analogous to the one for periodic points introduced in section 3). In fact, the following fact is equivalent to unique ergodicity: for every continuous function $g$ the ergodic average $(1 / T) \int_{0}^{T} g\left(\phi_{t} x\right) d t$ converges uniformly to $\int_{\mathbf{M}} g d \mu_{\text {Leb }}$. The usual proof of the equivalence of the two versions of unique ergodicity holds in the case of continuous maps (see, e.g., [W]). The uniform distribution of orbits version of 


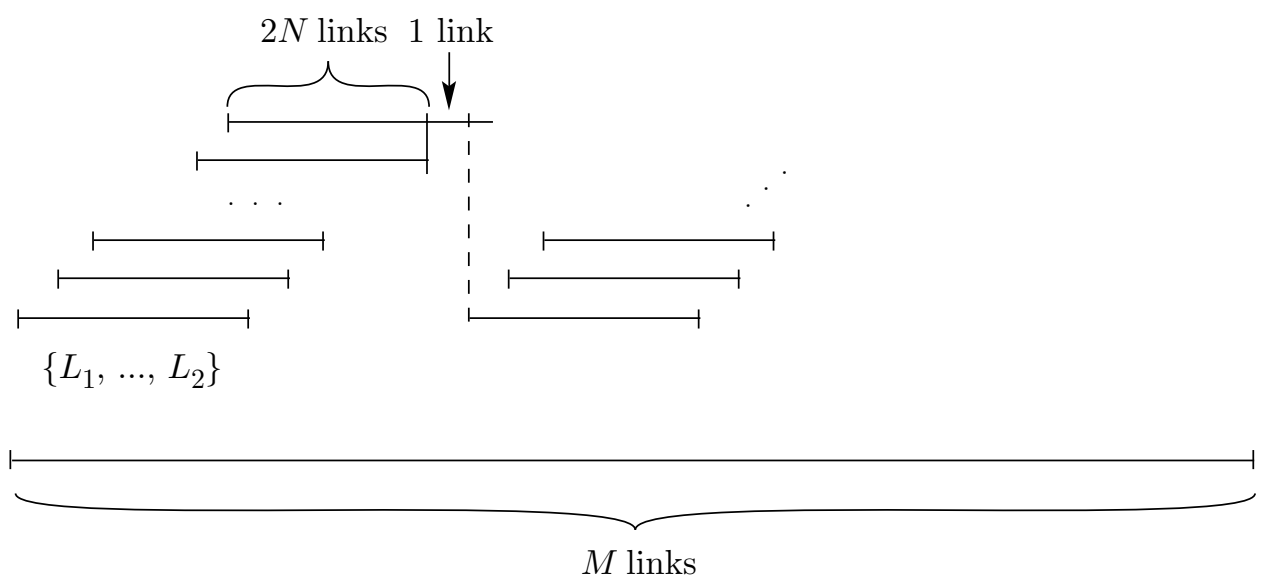

FIGURE 7. Covering the trajectory by well-distributed trajectory segments

unique ergodicity can be directly demonstrated for rational polygons by combining results of Masur [M1] and Boshernitzan [Bo1].

We also choose $N$ so large that, for any $k \geq N$, if the first $k$ links of the orbit of any point $x$ are $2 \varepsilon / 3$-well distributed, then the first $k+1$ links are $\varepsilon$-well distributed. The rest of the proof is similar to the proof of Theorem 1, so we indicate only which steps need to be modified. We choose $\delta:=\delta_{2 N}$ and the periodic point $\left(q_{0}, \varphi_{0}\right)$ so that it satisfies $\left|\varphi_{0}-\theta\right|<\delta_{2 N}$ and $\left|\varphi_{0}-\theta\right|<\varepsilon /(3 \cdot$ const $\cdot 2 N \cdot \operatorname{diam} Q)$. Then the periodic trajectory of $\left(q_{0}, \varphi_{0}\right)$ shadows the $\varepsilon / 3$-well distributed uniquely ergodic trajectory for $2 N$ links either forward or backward. Thus the first $2 N$ links of this orbit are $2 \varepsilon / 3$-well distributed and the first $2 N+1$ links are $\varepsilon$-well distributed. Suppose the number of links of the $\left(q_{0}, \varphi_{0}\right)$ trajectory is $M$. If $M \leq 2 N+1$, then the trajectory is clearly $\varepsilon$-well distributed.

If $M>2 N+1$, let the cyclically ordered set $\left\{L_{1}, L_{2}, \ldots, L_{M}\right\}$ denote the links of the trajectory. We want to partition the trajectory into $\varepsilon$-well distributed trajectory segments of lengths between $N$ and $2 N$. From formula $\left(1^{\prime}\right)$ it is clear that the concatenation of $\varepsilon$-well distributed trajectory segments is itself $\varepsilon$-well distributed. Therefore, the construction of such a partition will finish the proof.

We call a trajectory segment $\left\{L_{i}, L_{i+1}, \ldots, L_{i+M-1}\right\}$ of length $M \geq N \varepsilon$-good if it and all its ordered subsets $\left\{L_{j}, \ldots, L_{j+k-1}\right\}$ of length $k \geq N$ are at least $\varepsilon$ well distributed. Let $T(k)$ be the $k$ th return time of the orbit of $\left(q_{0}, \varphi_{0}\right)$ to the boundary $\partial Q$. To construct such a partition we start by covering the trajectory by ordered sets $\left\{L_{i}, \ldots, L_{i+2 N-1}\right\}$ of length $2 N$ that are $\varepsilon$-good. To do this, note that for each $k$ either the forward $2 N$ links of $\phi_{T(k)}\left(q_{0}, \varphi_{0}\right)$ are $2 \varepsilon / 3$-good or the backwards $2 N$ links are $2 \varepsilon / 3$-good. Without loss of generality we suppose that $\left\{L_{1}, \ldots L_{2 N}\right\}$ is $2 \varepsilon / 3$-good. Now consider the point $\phi_{T(2 N+1)}\left(q_{0}, \varphi_{0}\right)$ to conclude that either $\left\{L_{2}, \ldots, L_{2 N+1}\right\}$ or $\left\{L_{2 N+2}, \ldots, L_{4 N+1}\right\}$ is $2 \varepsilon / 3$ good. If the former occurs, then we have covered $\left\{L_{1}, \ldots, L_{2 N+1}\right\}$ by the $\varepsilon$-good sets $\left\{L_{1}, \ldots, L_{2 N}\right\}$ and $\left\{L_{2}, \ldots, L_{2 N+1}\right\}$. If the latter occurs, then the link $L_{2 N+1}$ is not covered. In this case we replace $\left\{L_{1}, \ldots, L_{2 N}\right\}$ by $\left\{L_{1}, \ldots, L_{2 N+1}\right\}$, which is $\varepsilon$-good since $\left\{L_{1}, \ldots, L_{2 N}\right\}$ was $2 \varepsilon / 3$-good. Thus we have covered $\left\{L_{1}, \ldots, L_{4 N+1}\right\}$ by the $\varepsilon$-good sets $\left\{L_{1}, \ldots, L_{2 N+1}\right\}$ and $\left\{L_{2 N+2}, \ldots, L_{4 N+1}\right\}$. Continuing this process inductively 
yields the desired covering (Fig. 7). To finish the proof we split the covering into a partition of $\varepsilon$-well distributed pieces of different lengths, but with all the lengths being at least $N$ and at most $2 N$.

Proof of Theorem 3. Fix a uniquely ergodic direction $\theta$. Let

$$
\begin{aligned}
A_{\varepsilon}^{\delta}(\theta):=\{q \in & Q \backslash V:(q, \varphi) \text { is an } \varepsilon \text {-well distributed } \\
& \text { periodic point for some } \varphi,|\varphi-\theta|<\delta\} .
\end{aligned}
$$

In the proof of Theorem 2 we showed that for all uniquely ergodic directions $\theta$ and for all $\varepsilon>0$ there is a $\delta:=\delta(\theta, \varepsilon)>0$ such that $A_{\varepsilon}^{\delta}(\theta)$ is dense in $Q$. The set $A_{\varepsilon}^{\delta}(\theta)$ is also open because each periodic point is contained in an open strip; that is, if $x=(q, \varphi)$ is periodic, then there is an open disc $D \subset Q \backslash V$ such that $\left(q^{\prime}, \varphi\right)$ is periodic for all $q^{\prime} \in D$ (see [GKT]). Choose a countable dense set of uniquely ergodic directions $\left\{\theta_{i}\right\} \subset \mathbf{S}^{1}$ and $\varepsilon_{i}>0$ satisfying $\lim _{i \rightarrow \infty} \varepsilon_{i}=0$. Furthermore choose positive numbers $\delta_{i} \leq \delta\left(\theta_{i}, \varepsilon_{i}\right)$ such that $\lim _{i \rightarrow \infty} \delta_{i}=0$. Then

$$
\bigcap_{i \in \mathbf{Z}^{+}} A_{\varepsilon_{i}}^{\delta_{i}}\left(\theta_{i}\right) \subset G
$$

and thus $G$ is residual.

Proof of Theorem 4. First we will show that the set $B$ is contained in a finite number of line segments. To do this we will consider only billiard trajectories which hit some side of the polygon perpendicularly. Let $\varphi_{i}$ be the direction perpendicular to the $i$ th side of $Q$. As discussed in section 2, the invariant surface $R_{\varphi_{i}}$ contains at most a finite number of generalized diagonals. Any perpendicular trajectory which enters a vertex is automatically a generalized diagonal, because its backward orbit also enters the same vertex. This means that only a finite number of points on the $i$ th side have singular perpendicular trajectories. The number of such diagonals is clearly less than the number of floors of the invariant surface $R_{\varphi_{i}}$ multiplied by the number of vertices of $Q$. We remark that the number of $R_{\varphi_{i}}$ 's floors is less than the greatest common denominator of all the inner angles of the polygon.

The perpendicular orbit through any other point on the $i$ th side hits that side perpendicularly twice and thus is periodic (for details see [Bo], [GSV]). For each point $q \in Q$ we consider the perpendiculars to the straight lines containing the sides of $Q$. Since $Q$ is convex, for the nearest side of $Q$, the base point of this perpendicular will belong to an interior point of the side and not to its continuation. Consequently, any point $q$ which is not covered by one of the finite number of generalized diagonals from the surfaces $\bigcup_{i} R_{\varphi_{i}}$ has a "perpendicular" periodic orbit passing through it.

We remark that by using the ideas from the proofs of Theorems 1, 2 and 3 we can prove a slightly stronger result. Namely, $B$ is contained in a Cantor subset of these segments.

If $Q$ is an acute or right triangle, then for each $q \in Q \backslash V$ the perpendicular to the lines containing the sides lie inside $Q$ (if $q \in \partial Q$, then this perpendicular is just the point $q$ ). For obtuse triangles the same is true for at least two sides. Thus for rational triangles, for each $q \in Q \backslash V$ there are at least two distinct singular perpendicular billiard orbits passing through $q$. Each point $q \in Q \backslash V$ which is not covered by a perpendicular periodic trajectory must be covered by two distinct perpendicular generalized diagonals. However, any two generalized diagonals which 
intersect do so transversely, or else they would coincide. If $d_{1}, \ldots, d_{k}$ denote the perpendicular generalized diagonals, then $B$ is contained in $\bigcup_{i \neq j}\left(d_{i} \cap d_{j}\right)$, a finite set.

Question. For which polygons are all $q \in Q \backslash V$ at least double covered by perpendicular trajectories? Note that this does not hold for the regular hexagon, although in this case it is easy to see that $B$ is empty.

Proof of Theorem 5. Let

$$
C_{\varepsilon}:=\left\{\varphi: \exists x \in R_{\varphi} \text { such that the orbit of } x \text { is not } \varepsilon \text {-dense }\right\} .
$$

If $R_{\varphi}$ contains no generalized diagonal, then the flow $\left.\phi\right|_{R_{\varphi}}$ is minimal, and thus every billiard orbit is dense. The set of generalized diagonals is at most countable, and thus so is $C_{\varepsilon}$.

The set $C_{\varepsilon}$ is clearly closed, for if $\varphi_{i} \in C_{\varepsilon}$ and $x_{i} \in R_{\varphi_{i}}$ is not an $\varepsilon$-well distributed point, then any weak limit point of the $x_{i}$ is also not an $\varepsilon$-well distributed point.

An example where $C_{\varepsilon}$ is countable is the following. We consider the L-shaped figure consisting of three identical squares as depicted in Figure 8(a). In this figure a periodic orbit with 4 links which avoids the right hand square is shown. In Figure 8 (b), an unfolding of length 3 of the L-shaped figure along with a periodic orbit with 8 links is drawn. This periodic orbit also never enters the right hand square.

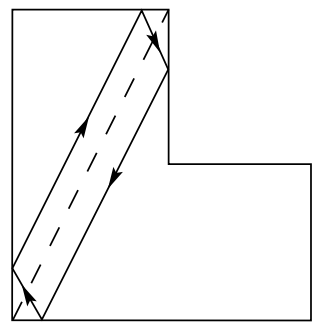

(a)

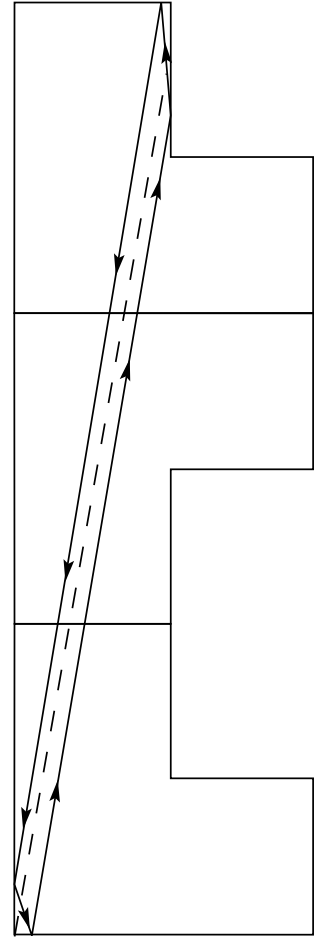

(b)

Figure 8. Periodic orbits in the L-shaped figure which do not enter the right hand square. 
In general, the analogous unfoldings of length $k$ contains a periodic orbit with $2 k+2$ links which avoids the right hand square.

Question. Are there convex examples of this phenomenon?

\section{REFERENCES}

[BKM] C. Boldrighini, M. Keane, and F. Marchetti, Billiards in polygons, Ann. Prob. 6 (1978), 532-540. MR 58:31007b

[Bo] M. Boshernitzan, Billiards and rational periodic directions in polygons, Amer. Math. Monthly 99 (1992), 522-529. MR 93d:51043

[Bo1] M. Boshernitza, A condition for minimal interval exchange maps to be uniquely ergodic, Duke Math. J. 52 (1985) 723-752. MR 87i:28012

[CFS] I.P. Cornfeld, S.V. Fomin and Ya.G. Sinai, Ergodic theory, Springer Verlag, Berlin, 1982. MR 87f: 28019

[GKT] G. Galperin, T. Krüger, and S. Troubetzkoy, Local instability of orbits in polygonal and polyhedral billiards, Comm. Math. Phys. 169 (1995), 463-473. MR 96d:58106

[GSV] G.A. Galperin, A.M. Stepin, and Ya.B. Vorobetz, Periodic billiard trajectories in polygons: generating mechanisms, Russian Math. Surv. 47:3 (1992), 5-80. MR 93h:58088

[Gu] E. Gutkin, Billiards in polygons, Physica D 19 (1986), 311-333. MR 87k:58085

[Gu1] E. Gutkin, Billiards on almost integrable polyhedral surfaces, Ergod. Th. Dyn. Sys. 4 (1984), 569-584. MR 86m:58123

[K] M. Keane, Interval exchange transformations, Math. Z. 141 (1975), 25-31. MR 50:10207

[KMS] S. Kerckhoff, H. Masur, and J. Smillie, Ergodicity of billiard flows and quadratic differentials, Annals Math. 124 (1986), 293-311. MR 88f:58122

[M] H. Masur, Closed trajectories for quadratic differentials with an application to billiards, Duke Math. J. 53 (1986), 307-313. MR 87j:30107

[M1] H. Masur, The growth rate of trajectories of a quadratic differential, Ergod. Th. Dyn. Sys. 10 (1990), 151-176. MR 91d:30042

[V] W. Veech, The billiard in a regular polygon Geom. Func. Anal. 2 (1992), 341-379. MR 94a: 11074

[V1] W. Veech, Teichmuller curves in moduli space, Eisenstein series and an application to triangular billiards, Invent. Math. 97 (1989), 553-583. MR 91h:58083a

[W] P. Walters, An introduction to ergodic theory, Springer-Verlag, 1982. MR 84e:28017

Department of Mathematics, Rice University, Houston, Texas 77005

E-mail address: michael@math.rice.edu

Forschungszentrum BiBos, Universität Bielefeld, Bielefeld, Germany

Current address: Department of Mathematics, Eastern Illinois University

E-mail address: cfgg@eiu.edu

Forschungszentrum BiBos, Universität Bielefeld, Bielefeld, Germany

Forschungszentrum BiBos, Universität Bielefeld, Bielefeld, Germany and Institute for Mathematical Science, SUNy at Stony Brook, Stony Brook, New York 11794

Current address: Department of Mathematics, University of Alabama at Birmingham, Birmingham, Alabama 35294

E-mail address: troubetz@math.uab.edu 